\title{
Role of residual solvents in the formation of volatile compounds after radiosterilisation of cefotaxime
}

\author{
N. Barbarin *, B. Rollmann, B. Tilquin \\ Universite Catholique De Louvain, Unité d'analyse chimique et physico-chimique des médicaments, CHAM 72.30, 72, \\ Avenue E. Mounier, B-1200 Brussels, Belgium
}

Received 19 August 1998; received in revised form 28 October 1998; accepted 3 November 1998

\begin{abstract}
Radiation sterilisation is a promising method to sterilise pharmaceutical products. However, this process is accompanied by a modification of odour due to volatile compounds formation. The origin of malodorous compounds produced during solid cefotaxime radiosterilisation has been investigated and several mechanisms are proposed to explain their appearance. Moreover, some quantitative data are given. Analysis of the degradation products was performed using a GC-ITD system with an injection by the static headspace technique. It appeared that some of the radio-induced compounds (such as carbon oxide sulfide and carbon disulfide) came from the degradation of the drug itself, whereas the formation of others required the presence of residual solvents which are volatile impurities already present before irradiation. Acetaldehyde directly came from impurities but the appearance of esters and acetaldehyde $O$-methyloxime was due to the presence of both cefotaxime and residual solvents together. Thus, the residual solvents play a key role in the radiolysis compounds formation (six of eight require the presence of them) and consequently in the modification of odour as well. (C) 1999 Elsevier Science B.V. All rights reserved.
\end{abstract}

Keywords: Radiosterilisation; Cefotaxime; Residual solvent; Odour modification; Headspace; Radiolysis

\section{Introduction}

The radiosterilisation of heat sensitive devices such as catheters, syringes, sutures, etc. was introduced about 25 years ago and is now widely used in many countries. The application of radiation to

\footnotetext{
* Corresponding author. E-mail: barbarin@cham.ucl.ac.be.
}

sterilise pharmaceuticals has increased in the last few years (Jacobs, 1995).

The considerable interest to use $\gamma$-rays for drug sterilisation arises from their high penetrating power and their isothermal character (Tilquin, 1991): the drug can be sterilised in its final package without any rise in temperature (Jacobs, 1985) and is thus particularly suitable for heat sensitive 
drugs like cephalosporins which cannot be sterilised by traditional methods like dry heat or steam sterilisation.

The dose required for radiation treatment of pharmaceuticals depends on the initial bioburden and on the radiosensitivity of the present species. The dose that we have chosen in this work (25 $\mathrm{kGy}$ ) is the one generally accepted as required for drug sterilisation. However, several pharmaceuticals can be sterilised using a lower dose (Jacobs and Wills, 1988; Dam et al., 1990; Whitby, 1993; Dam et al., 1996) which has the advantage of minimizing damage of the products (Gopal, 1978).

Cefotaxime is an antibiotic that belongs to the cephalosporins group. Its molecular structure is presented in Fig. 1. As for other drugs, its irradiation does not modify its activity (Zegota et al., 1994, 1995; Basly et al., 1997) but causes colour and odour modifications. These modifications are due to the production of new compounds of radiolytic origin (Zeegers et al., 1993; Varshney and Patel, 1994), and which are potentially toxic (Schüttler and Bögl, 1993a,b, 1994). The odour detected in irradiated cefotaxime is related to the production of several malodorous compounds like acetaldehyde or esters (Barbarin et al., 1996).

In a former study (Barbarin et al., 1996), two hypotheses were advanced to explain the origin of the radio-induced volatile compounds. They could come either from the molecular degradation of the drug itself, or, because of the nature of some radiolytic products (esters), from the residual solvents present in the non-irradiated drug, particularly methanol, ethanol and acetic acid.

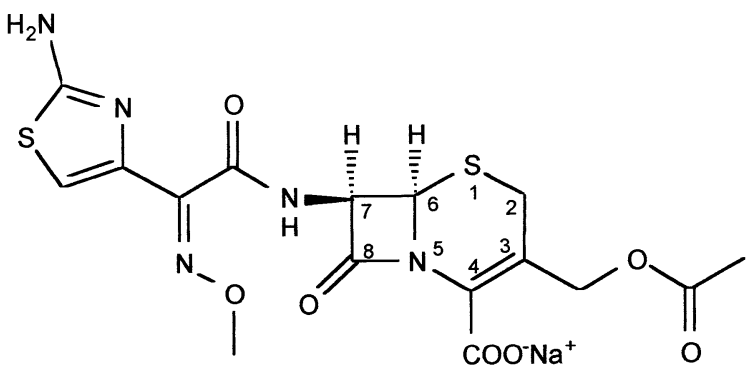

Fig. 1. Molecular structure of cefotaxime sodium salt.
The aim of this study is to answer to questions previously formulated: to find out the origin of the volatile compounds and to understand the influence of the residual solvents. For that purpose, a headspace GC system with an ion trap detector was used.

Moreover, in order to have an idea of the amount of analytes present or produced by irradiation, some quantitative results are given. Quantification for three volatile compounds: one residual solvent (ethanol) and two radiolysis products (acetaldehyde and formic acid ethyl ester) was performed.

\section{Materials and methods}

Cefotaxime sodium salt $\left(\right.$ Claforan $\left.^{\circledR}\right)$ was kindly provided by Hoechst. Silicagel $60 \AA$, ethanol and propan-1-ol were supplied by Merck. Methanol and acetic acid were supplied by UCB.

\subsection{Qualitative experiments}

One gram of solid cefotaxime or silicagel was irradiated in a 10-ml vial, sealed with a rubber septum with a crimp cap, in the panoramic ${ }^{60} \mathrm{Co}$ chamber in Louvain-la Neuve (Belgium) at a dose of $25 \mathrm{kGy}$ under an atmosphere of air. The absorbed dose was measured using the Fricke dosimeter. The dose rate was $0.23 \mathrm{~Gy} \mathrm{~s}^{-1}$.

A headspace GC system with an ion trap detector was employed for volatile compounds analysis. This system consisted of an HP 19395A static headspace sampler, an HP 5890 GC and an ITD from Finnigan Mat. The HP 19395A settings were as follows: probe down at $1 \mathrm{~s}$; pressurize vial, $3-15 \mathrm{~s}$; vent/fill loop, $14-16 \mathrm{~s}$; inject $18 \mathrm{~s}$ to 2 min; probe up at $3 \mathrm{~min}$; carrier gas pressure, about 1.2 bar; auxiliary gas pressure, about 1.2 bar; servo air pressure, about 4 bar; bath temperature, $45^{\circ} \mathrm{C}$; vent/fill loop temperature, $50^{\circ} \mathrm{C}$. The samples were heated for $1 \mathrm{~h}$. The GC conditions were as follows: Pora Plot Q column of $25 \mathrm{~m} \times$ $0.32 \mu \mathrm{m}$ i.d. from Chrompack. The carrier gas used was helium purified with an oxygen and a moisture trap, at a flow rate of $1.9 \mathrm{ml} / \mathrm{min}$. The injection port temperature was set at $250^{\circ} \mathrm{C}$ and 
the oven temperature at $40^{\circ} \mathrm{C}$ for $5 \mathrm{~min}$ and then heated at $5^{\circ} \mathrm{C} / \mathrm{min}$ to $200^{\circ} \mathrm{C}$. This temperature programme did not enable the derivative of propan-1-ol to be eluted in a reasonable time, so another temperature programme was used for the experiments with propan-1-ol: the oven temperature was set at $40^{\circ} \mathrm{C}$, then directly heated at $5^{\circ} \mathrm{C} / \mathrm{min}$ to $210^{\circ} \mathrm{C}$ and finally held for $5 \mathrm{~min}$ at $210^{\circ} \mathrm{C}$. The ion trap detector was used in the full scan mode. The scan range was set from $m / z 30$ to 150.

Silicagel was used to adsorb vapours of methanol, ethanol and acetic acid in order to irradiate them without cefotaxime. The exposure time of silicagel to vapours was chosen to have more or less the same peak area and the same peak ratio as in cefotaxime. The exposure was made in two steps. The first step consisted of exposing silicagel to vapours of acetic acid for $30 \mathrm{~s}$ and then airing it for $90 \mathrm{~min}$ on a bench at ambient temperature. The second step consisted of exposing silicagel to vapours of methanol and ethanol for 5 min and airing it for $90 \mathrm{~min}$ under the same conditions.

\subsection{Quantitative experiments}

Another method was used for quantification. The same headspace GC system was used with detection by FID. The samples of cefotaxime were irradiated in the solid state and then dissolved just before analysis in an aqueous solution of $\mathrm{NaCl} 5 \%$ $(\mathrm{w} / \mathrm{v})$ containing various amounts of standard in order to perform the method of standard additions. This permitted us to eliminate any possible drug matrix effect.

\subsubsection{Samples and standards preparation}

The standard solutions were prepared by partially filling a $100-\mathrm{ml}$ volumetric flask with an aqueous solution of $\mathrm{NaCl} 5 \%(\mathrm{w} / \mathrm{v})$, weighing it with the stopper, adding various amounts of standards and weighing it again after replacing the stopper. This solution was then diluted to $100 \mathrm{ml}$ with the aqueous solution of $\mathrm{NaCl}$ (solution $\mathrm{A}$ ); 1 $\mathrm{ml}$ of solution A was diluted to $250 \mathrm{ml}$ with the same solution of $\mathrm{NaCl}$ (solution $\mathrm{B}$ ).
For the quantification of each analyte, four different standard solutions B were made, each containing a different amount of standard.

The sample solutions consisted of $200 \mathrm{mg}$ of cefotaxime in a $10-\mathrm{ml}$ vial, irradiated at a dose of $25 \mathrm{kGy}$ and which were dissolved just before analysis by adding $1 \mathrm{ml}$ standard solutions $\mathrm{B}$.

\section{Results and discussion}

The total response chromatograms of the (a) non-irradiated and (b) irradiated cefotaxime are presented in Fig. 2. Some peaks are already present before irradiation and correspond to residual solvents (such as methanol, ethanol, tetrahydrofuran, etc.). They are designated by letters. Others appear with irradiation and correspond to radio-induced compounds (such as carbon oxide sulfide, acetaldehyde or formic acid methyl ester for instance). They are designated by numbers. These results were previously discussed (Barbarin et al., 1996). The peaks' identity is listed in Tables 1 and 2.

\subsection{Irradiation of cefotaxime in the absence of the residual solvents}

The same experiment was performed after elimination of the residual solvents: $1 \mathrm{~g}$ of powder was put under a vacuum of $10^{-2}$ torr for $24 \mathrm{~h}$ and then analysed. The total response chromatogram is presented in Fig. 3a. This preliminary treatment eliminated the residual solvents. Only a small peak of ethanol remained (peak b), the most abundant impurity. Fig. 3b showed the total response chromatogram of cefotaxime, irradiated after vacuum treatment. Carbon oxide sulfide (peak no. 1) and carbon disulfide (peak no. 5) were still detected in similar concentrations to the first experiment (cf. Fig. 2). Acetaldehyde (peak no. 2), was still detected but at a very low concentration. Fig. $3 \mathrm{~b}$ also showed the appearance of methanol (peak a) and the increase of ethanol (peak b). On the other hand, formic acid methyl ester (peak no. 3); formic acid ethyl ester (peak no. 6); acetic acid methyl ester (peak no. 7) and acetaldehyde $O$-methyloxime (peak no. 8) were not detected. 


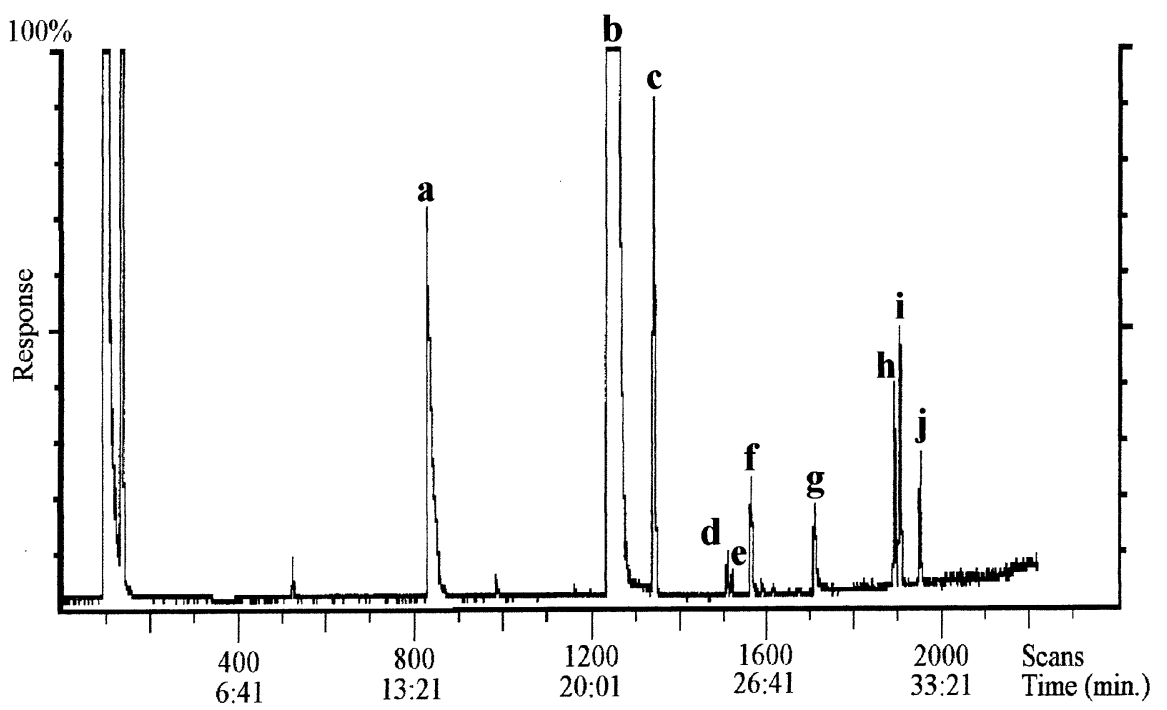

(a) non-irradiated cefotaxime

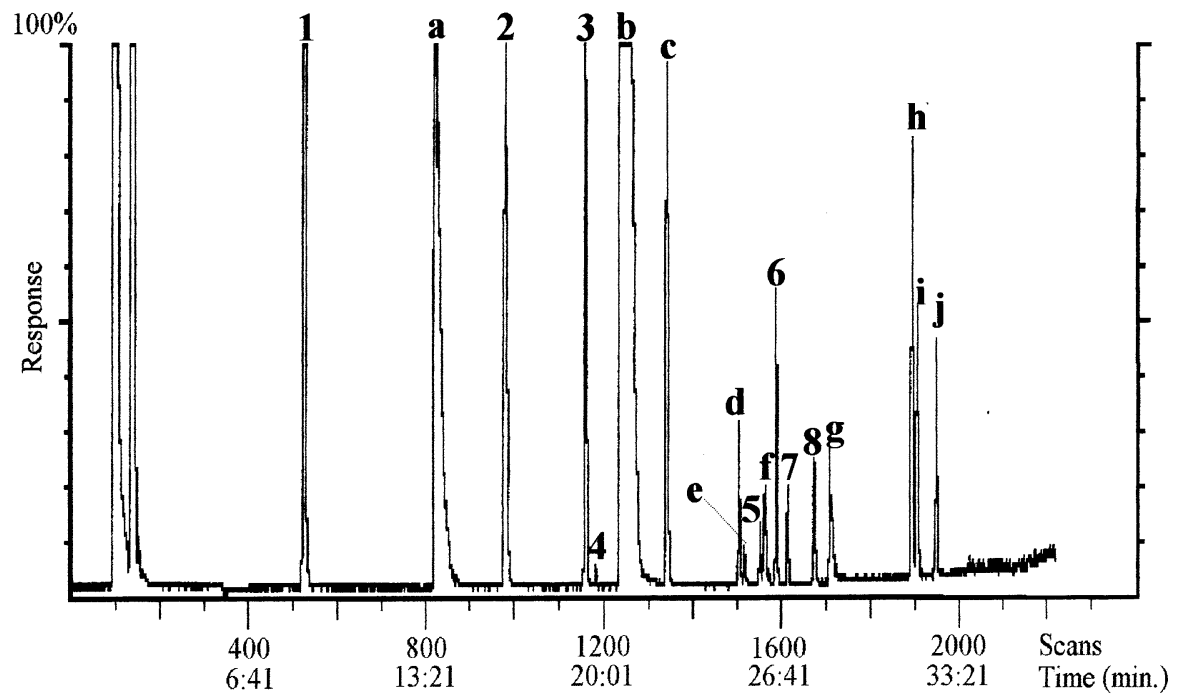

(b) irradiated cefotaxime

Fig. 2. Total response chromatogram of (a) non-irradiated and (b) irradiated cefotaxime sodium salt. Temperature programme: $40^{\circ} \mathrm{C}$ for $5 \mathrm{~min} ; 5^{\circ} \mathrm{C} / \mathrm{min}$ to $200^{\circ} \mathrm{C}$.

Consequently, of the eight volatile compounds produced by irradiation, only two (carbon oxide sulfide and carbon disulfide) were not affected by the elimination of the residual solvents. These should come from the degradation of the drug, in particular from those parts of cefotaxime containing sulphur atoms. On the other hand, the concentration of the others decreased (acetaldehyde) or they even disappeared (the esters and acetaldehyde $O$-methyloxime) indicating the fun- 
Table 1

List of the volatile impurities found in the non-irradiated cefotaxime

\begin{tabular}{lll}
\hline Peak & Identification & Retention time (min) \\
\hline a & Methanol & 13.38 \\
b & Ethanol & 20.34 \\
c & Acetonitrile & 22.19 \\
d & Acetone & 25.07 \\
e & Dichloromethane & 25.18 \\
f & Isopropanol & 26.02 \\
g & Acetic acid & 28.31 \\
h & Unidentified & 31.31 \\
I & Tetrahydrofuran & 31.43 \\
j & Acetic acid ethyl ester & 32.27 \\
\hline
\end{tabular}

damental role of the residual solvents in their formation.

The appearance of methanol and the increase of ethanol could have two causes. They could be produced in very low concentrations by the irradiation, or the radiation could help the desorption of small quantities of these compounds not completely eliminated by the vacuum.

\subsection{Irradiation of the residual solvents without cefotaxime}

Two hypotheses could be advanced to explain the role of the residual solvents in the radio-induced volatile compounds formation:

1. These could directly come from the volatile impurities: acetaldehyde might be derived

Table 2

List of the volatile compounds produced by irradiation of cefotaxime

\begin{tabular}{lll}
\hline Peak no. & Identification & $\begin{array}{l}\text { Retention time } \\
(\mathrm{min})\end{array}$ \\
\hline 1 & Carbon oxide sulfide & 8.40 \\
2 & Acetaldehyde & 16.19 \\
3 & Formic acid methyl ester & 19.17 \\
4 & Unidentified & 19.40 \\
5 & Carbon disulfide & 25.52 \\
6 & Formic acid ethyl ester & 26.29 \\
7 & Acetic acid methyl ester & 26.56 \\
8 & Acetaldehyde $O$-methyl- & 27.54 \\
& oxime & \\
\hline
\end{tabular}

from ethanol; methyl and ethyl esters might be formed by radicals produced in methanol, ethanol and acetic acid.

2. They could be formed by reaction between radicals coming from cefotaxime with the residual solvents.

To discriminate between these two hypotheses, the residual solvents suspected of having a role in the formation of the volatile compounds were irradiated in the absence of cefotaxime. For that purpose, $1 \mathrm{~g}$ of silicagel was exposed to vapours of acetic acid, methanol and ethanol in order to adsorb them on the silicagel.

Fig. 4a showed the total response chromatogram of the non-irradiated silicagel exposed to vapours. Methanol (peak a), ethanol (peak b) and acetic acid (peak g) were well present. Extra peaks appeared after irradiation (Fig. 4b):

1. Carbon oxide sulfide (peak no. 1) appeared at low concentration.

2. Acetaldehyde (peak no. 2) was detected in similar concentration in the presence (Fig. 2) or absence (Fig. 4) of cefotaxime.

3. Formic acid methyl ester (peak no. 3) was detected in much lower concentration than in the presence of cefotaxime.

4. The other esters and acetaldehyde $O$-methyloxime were not detected.

These results showed that only acetaldehyde directly came from the residual solvents, probably from ethanol. On the other hand, the formation of the other compounds required the presence of both the residual solvents and cefotaxime and were formed by reaction of radicals coming from the drug with the residual solvents (even if formic acid methyl ester was formed at very low concentration in the absence of cefotaxime). The small peak of carbon oxide sulfide came from the rubber septum of the vial: a small peak was indeed observed after the irradiation of an empty vial. Thus, if the main quantity of COS produced by irradiation of cefotaxime was due to its degradation, a very small amount could be attributed to the irradiated septum.

A mechanism can be proposed to explain the formation of the volatile compounds. Radicals formed in cefotaxime could react with the residual solvents to give stable and volatile compounds. A 


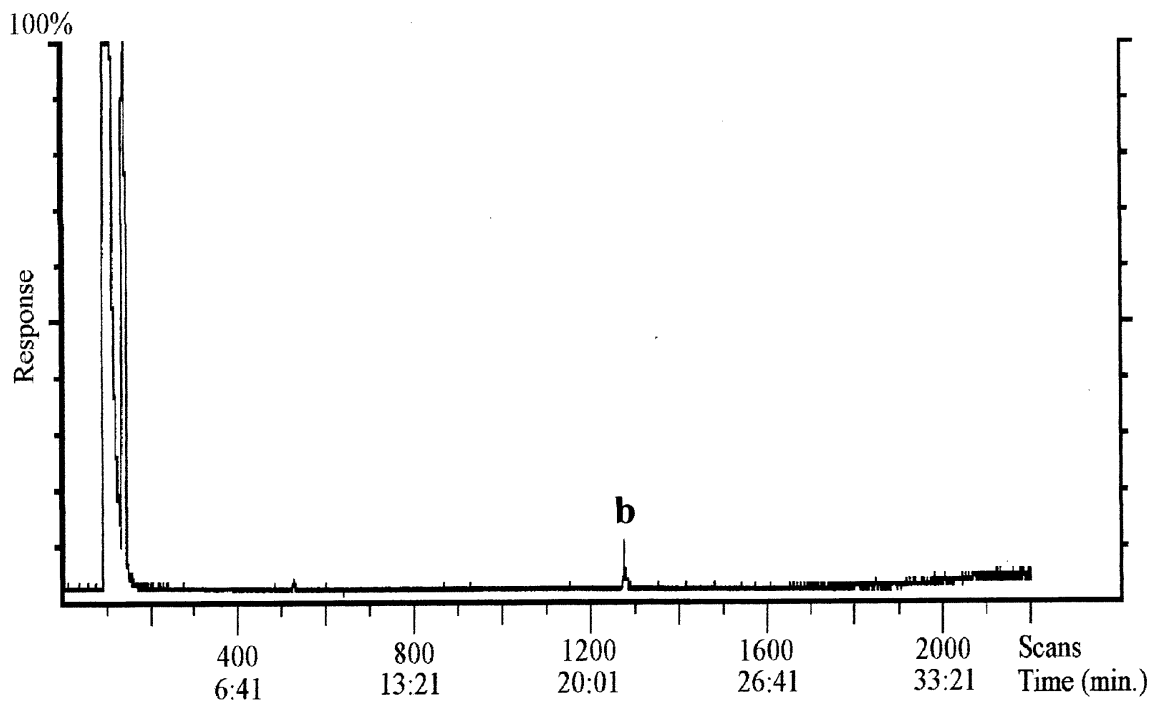

(a) non-irradiated cefotaxime

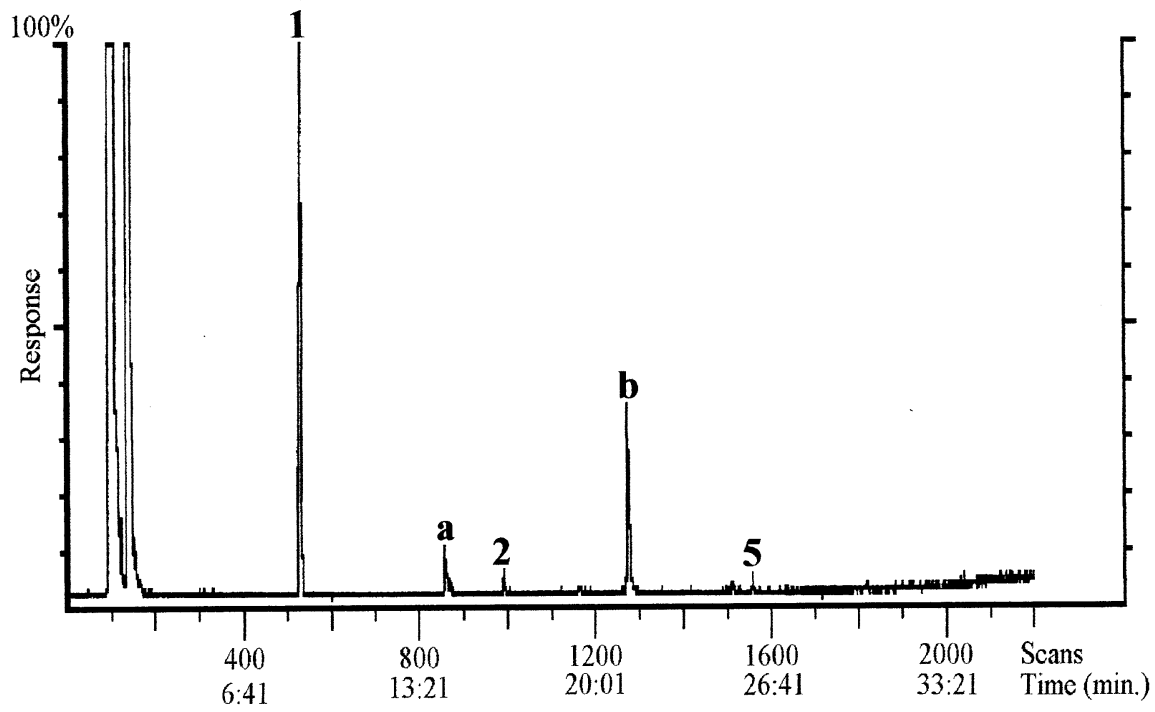

(b) irradiated cefotaxime

Fig. 3. Total response chromatogram of (a) non-irradiated and (b) irradiated cefotaxime sodium salt after vacuum treatment. Temperature programme: $40^{\circ} \mathrm{C}$ for $5 \mathrm{~min} ; 5^{\circ} \mathrm{C} / \mathrm{min}$ to $200^{\circ} \mathrm{C}$.

formyl radical, for instance, could react with methanol and ethanol to give the formic acid methyl ester and the formic acid ethyl ester, respectively. An acetyl radical could also be formed in cefotaxime and react with methanol to give acetic acid methyl ester. This radical could also react with ethanol to give acetic acid ethyl ester but since this compound is already present as a residual solvent, it could not be detected as a radiolysis compound. 


\subsection{Irradiation of cefotaxime with propan-1-ol}

To support this hypothesis, the residual solvents of cefotaxime were replaced by propan- 1-ol. For that purpose, the impurities were first eliminated by exposition of cefotaxime to vacuum and then $20 \mu \mathrm{l}$ of propan-1-ol were added.

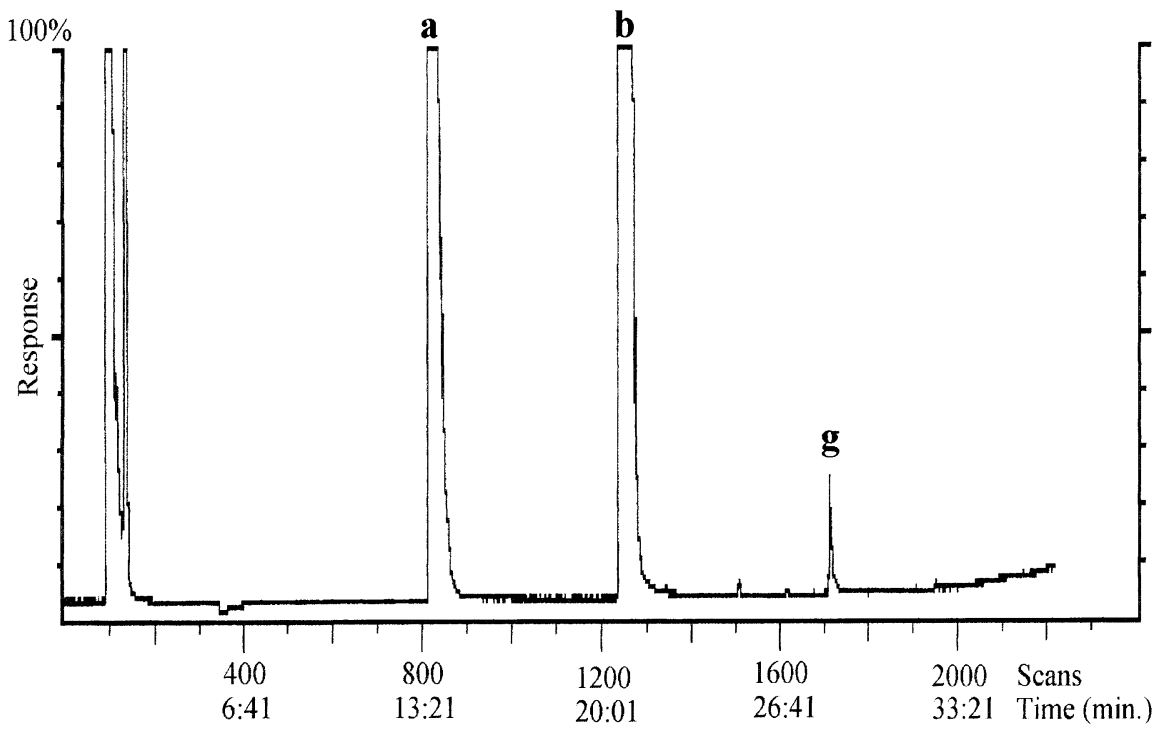

(a) non-irradiated silicagel

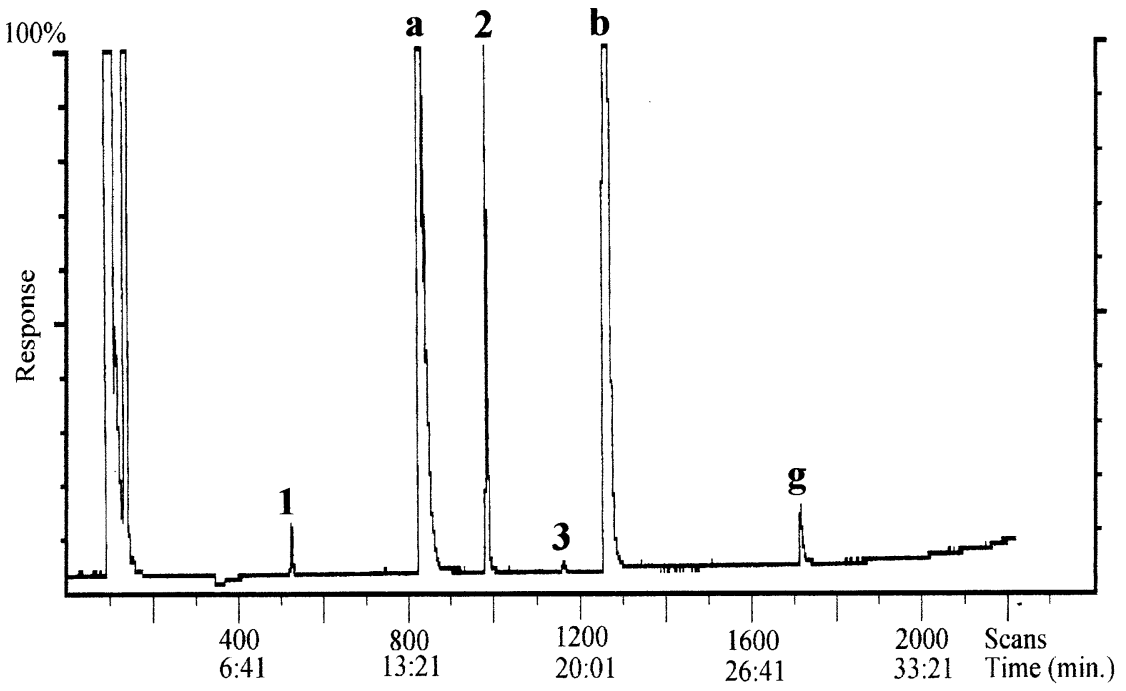

(b) irradiated silicagel

Fig. 4. Total response chromatogram of (a) non-irradiated and (b) irradiated silicagel. Temperature programme: $40^{\circ} \mathrm{C}$ for 5 min; $5^{\circ} \mathrm{C} / \mathrm{min}$ to $200^{\circ} \mathrm{C}$. 


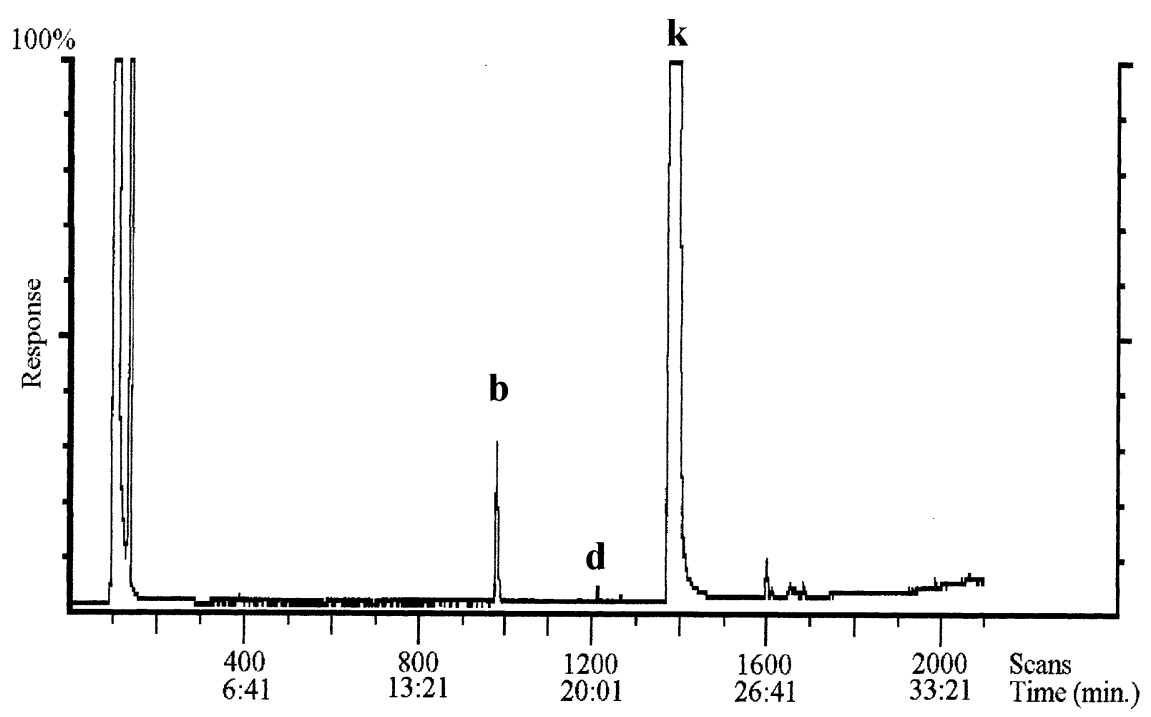

(a) non-irradiated cefotaxime

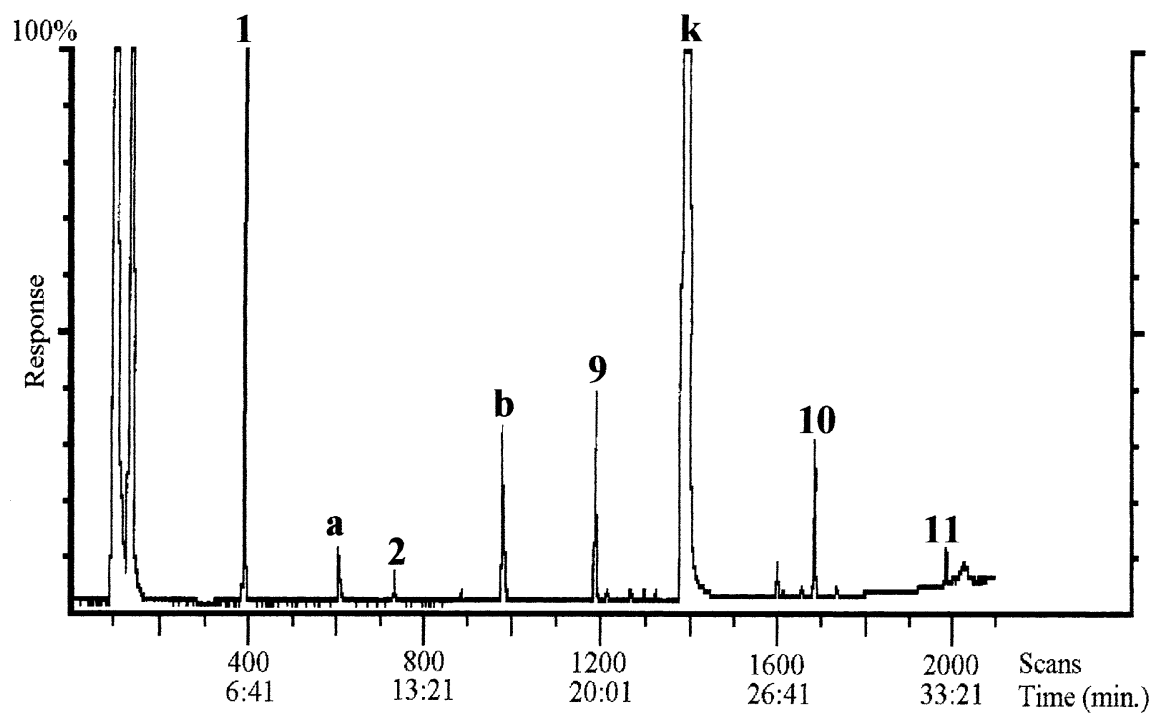

(b) irradiated cefotaxime

Fig. 5. Total response chromatogram of (a) non-irradiated and (b) irradiated cefotaxime sodium salt exposed to vacuum and where propan-1-ol was added. Temperature programme: $40^{\circ} \mathrm{C}$ for $0 \mathrm{~min} ; 5^{\circ} \mathrm{C} / \mathrm{min}$ to $210^{\circ} \mathrm{C} ; 210^{\circ} \mathrm{C}$ for $5 \mathrm{~min}$.

Fig. 5a showed the total response chromatogram of the non-irradiated sample. A peak of propan-1-ol (peak $\mathrm{k}$ ) was detected. A small quantity of ethanol (peak b) was not completely eliminated. Acetone (peak d) is an impurity of propan-1-ol. The irradiated sample showed extra peaks (cf. Fig. 5b). Carbon oxide sulfide (peak no. 1) was still present, and the small quantity of 
acetaldehyde (peak no. 2) could be explained by the presence of residual ethanol. But three new peaks appeared and were identified as propanal (peak no. 9), formic acid propyl ester (peak no. 10) and acetic acid propyl ester (peak no. 11). Propanal could directly come from propan-1-ol as acetaldehyde came from ethanol. The formyl and acetyl radicals could be formed in cefotaxime and react with propan-1-ol to give the two propyl esters.

As the same results were obtained from other cephalosporins with other lateral chains (unpublished data), the cephem ring might be at the origin of these formyl and acetyl radicals which could then react with the alcohol's to give the esters as illustrated by the following reactions:

$(\mathrm{HCO})^{\circ}+\mathrm{CH}_{3} \mathrm{OH} \rightarrow \mathrm{HCOOCH}_{3}+\mathrm{H}^{\circ}$

$(\mathrm{HCO})^{\circ}+\mathrm{CH}_{3} \mathrm{CH}_{2} \mathrm{OH} \rightarrow \mathrm{HCOOCH}_{2} \mathrm{CH}_{3}+\mathrm{H}^{\circ}$

These reactions are only hypothetical and others such as ionic reactions could be involved.

\subsection{Quantification}

The analysis method used for qualitative experiments did not permit us to have acceptable quantitative data. The main reasons for this were the quite non linear response of the ion trap detector and the poorly reproducible equilibration between the solid and the vapour phase during the headspace equilibrium. Another method was used for quantification. Reproducible data were obtained using a flame ionisation detector and an equilibrium between a liquid and a vapour phase. The use of an aqueous solution of $\mathrm{NaCl} 5 \%(\mathrm{w} / \mathrm{v})$ to dissolve cefotaxime, permitted us to perform a 'salting out' effect in order to increase the sensitivity of the detection.
Drug matrix effects can influence the activity of a volatile analyte. To avoid these effects, we used the standard additions method for quantification. In this method, a sample was analysed alone (dissolution by $1 \mathrm{ml}$ of the aqueous solution of $\mathrm{NaCl}$ ) or after addition of the standard solutions $\mathrm{B}$ containing the four incremental levels of standards.

The calibration curve was obtained by plotting the peak of analyte area on the ordinate against the amount of standard added (in ppm). A calibration curve was thus obtained for each volatile compound. The amount of analyte was determined by dividing the $y$-intercept of the fitted line by the slope. The results are presented in Table 3.

From Table 3, we determined the radiation yields defined as the ' $\mathrm{G}$ values' for the two radiolysis compounds, where $G(X)$ is the amount of moles of a species $X$ produced per joule absorbed. $G$ (formic acid ethyl ester) $=5.29 \times 10^{-9} \mathrm{~mol} / \mathrm{J}$ $G($ acetaldehyde $)=5.84 \times 10^{-5} \mathrm{~mol} / \mathrm{J}$

The interaction between ionizing radiations and a medium is function of the density of electrons. The molecules of drug, richer in electrons and more numerous (the purity of cefotaxime was higher than 96\%) receive almost all the energy. Radicals can be formed in cefotaxime and could react with the volatile impurities to give stable and volatile compounds but the energy could also propagate among the crystalline matrix and could activate molecules of impurities (Tilquin, 1985) which can give new compounds (ethanol gives acetaldehyde) as indicated by the much higher $G$ value of acetaldehyde in comparison with that of formic acid ethyl ester.

The formation of acetaldehyde $O$-methyloxime also depends on the presence of residual solvents.

Table 3

Quantitative results

\begin{tabular}{lccc}
\hline & Ethanol & Acetaldehyde & Formic acid ethyl ester \\
\hline Number of experimental data points & 20 & 20 & 20 \\
Regression & $y=0.634 x+493$ & $y=2.36 x+118$ & $y=9.48 x+93$ \\
$R^{2}$ & 0.9995 & 0.9960 & 0.9995 \\
S.D. of the slope & 0.008 & 0.07 & 0.09 \\
S.D. of the constant & 8 & 3 & 2 \\
Amount detected (ppm) & $778 \pm 16$ & $50 \pm 2$ & $9.8 \pm 0.3$ \\
\hline
\end{tabular}


A possible mechanism would be an activation by the irradiation of the oxime group of cefotaxime. This group is known to be particularly sensitive to $\gamma$-rays (Crucq and Tilquin, 1996a,b). A reaction between this activated group and acetaldehyde could give acetaldehyde $O$-methyloxime. Further experiments will have to be made to verify this hypothesis and to propose other degradation pathways.

\section{Conclusions}

Our results demonstrate that residual solvents play a key role in radiolysis compounds formation. Among the eight products detected, only two (carbon oxide sulfide and carbon disulfide) are formed in their absence. The residual solvents are necessary to observe all the others. Acetaldehyde comes from the irradiation of ethanol. The formation of the esters and acetaldehyde $O$-methyloxime requires both residual solvents and cefotaxime. Consequently, they come from an interaction between the solvents and the drug. The esters could come from a reaction between formyl or acetyl radicals with methanol and ethanol.

Thus, the modification of odour and the possible toxicity, due to the volatile compounds, also depends on the presence of residual solvents. They could be reduced, at least in part, by eliminating the volatile impurities.

\section{References}

Barbarin, N., Crucq, A.S., Tilquin, B., 1996. Study of volatile compounds from the radiosterilization of solid cephalosporins. Radiat. Phys. Chem. 48, 787-794.

Basly, J.P., Longy, I., Bernard, M., 1997. Radiation sterilization of formoterol. Pharm. Res. 14, 810-814.

Crucq, A.S., Tilquin, B., 1996. Attack of cefotaxime by different radicals: comparison of the effects. Free Radic. Biol.
Med. 21, 827-832.

Crucq, A.S., Tilquin, B., 1996. Method to identify products induced by radiosterilization. A study of cefotaxime sodium. J. Pharm. Belg. 51, 285-288.

Dam, A., Gazso, L.G., Grigorova, P., 1990. Microbiological investigations for radiation treatment of pharmaceuticals. Radiat. Phys. Chem. 35, 396-399.

Dam, A., Gazso, G., Kaewpila, S., Mascheki, 1996. Radiation treatment of pharmaceuticals. Radiat. Phys. Chem. 47, 515-517.

Gopal, N.G.S., 1978. Radiation sterilization of pharmaceuticals and polymers. Radiat. Phys. Chem. 12, 35-50.

Jacobs, G.P., 1985. Current trends in the radiosterilization of pharmaceuticals. Isr. Pharm. J. 25, 25-30.

Jacobs, G.P., 1995. A review of the effects of gamma radiation on pharmaceuticals materials. J. Biomed. Appl. 10, 59-96.

Jacobs, G.P., Wills, P.A., 1988. Recent developments in the radiation sterilization of pharmaceuticals. Radiat. Phys. Chem. 31, 685-691.

Schüttler, C., Bögl, K.W., 1993. Influence of radiation treatment on pharmaceuticals: a review. Part 1: Morphine derivatives and others alkaloids. J. Radiat. Steriliz. 1, 43-82.

Schüttler, C., Bögl, K.W., 1993. Influence of radiation treatment on pharmaceuticals: a review. Part 2: Antibiotics. J. Radiat. Steriliz. 1, 229-262.

Schüttler, C., Bögl, K.W., 1994. Influence of radiation treatment on pharmaceuticals: a review. Part 3: Penicillins. J. Radiat. Steriliz. 1, 327-345.

Tilquin, B., 1985. Contribution radicalaire à la radiolyse des alcanes à $77 \mathrm{~K}$. Ciaco, Louvain-la-Neuve.

Tilquin, B., 1991. Radio-stérilisation de médicaments. J. Pharm. Belg. 46, 396-398.

Varshney, L., Patel, K.M., 1994. Effects of ionizing radiations on a pharmaceutical compound, chloramphenicol. Radiat. Phys. Chem. 43, 471-480.

Whitby, J.L., 1993. Microbial aspects relating to the choice of radiation sterilization dose. Radiat. Phys. Chem. 42, 577580

Zeegers, F., Crucq, A.S., Gibella, M., Tilquin, B., 1993. Radiolyse et radiostérilisation des médicaments. J. Chem. Phys. 90, 1029-1040.

Zegota, H., Koprowski, M., Zegota, A., 1994. Stability of cefuroxime following gamma-irradiation in the solid state. Radiat. Phys. Chem. 43, 343-348.

Zegota, H., Koprowski, M., Zegota, A., 1995. Effect of gamma-irradiation on cefotaxime in the solid state. Radiat. Phys. Chem. 45, 223-229. 